\title{
Genome-wide analysis of DNA methylation identifies the apoptosis-related gene UQCRH in renal cancer
}

\section{Kosuke Miyakuni}

The University of Tokyo: Tokyo Daigaku

Jun Nishida

The University of Tokyo: Tokyo Daigaku

\section{Daizo Koinuma}

The University of Tokyo: Tokyo Daigaku

\section{Genta Nagae}

The University of Tokyo: Tokyo Daigaku

\section{Hiroyuki Aburatani}

The University of Tokyo: Tokyo Daigaku

\section{Kohei Miyazono}

The University of Tokyo: Tokyo Daigaku

Shogo Ehata ( $\nabla$ ehata-jun@umin.ac.jp )

The University of Tokyo: Tokyo Daigaku https://orcid.org/0000-0002-6740-9391

\section{Research}

Keywords: Renal cancer, apoptosis, DNA methylation, UQCRH

Posted Date: January 19th, 2021

DOl: https://doi.org/10.21203/rs.3.rs-147857/v1

License: (9) This work is licensed under a Creative Commons Attribution 4.0 International License. Read Full License 


\section{Abstract \\ Background}

DNA hypermethylation is frequently observed in clear cell renal cell carcinoma (ccRCC) and correlates with poor clinical outcomes. However, the detailed function is not fully uncovered.

\section{Methods}

A target for DNA methyltransferases (DNMTs) was explored in ccRCC cells. Highly malignant derivatives of human ccRCC cells were established via serial orthotopic inoculation in mice. Then, DNA methylated sites were genome-widely analyzed using methylation array in reference to RNA-sequencing data.

\section{Results}

We found that $D N M T 3 B$ upregulation contributes to renal cancer progression and identified the ubiquinol cytochrome $c$ reductase hinge protein (UQCRH) as a methylation target in advanced ccRCC. The expression of UQCRH in human cCRCC tissues was lower than that in normal adjacent tissues. Furthermore, its silencing attenuated cytochrome $c$ release in response to apoptotic stimuli, resulting in enhanced primary tumor formation in vivo. Moreover, DNA demethylation enhanced the therapeutic efficiency of the mammalian target of rapamycin (mTOR) inhibitor everolimus in vivo.

\section{Conclusions}

These findings suggest that the DNMT3B-induced UQCRH methylation may contribute to renal cancer progression and that DNMT inhibitors exhibit potential clinical significance for CcRCC treatment.

\section{Background}

Renal cell carcinoma (RCC) causes more than 100,000 deaths world-wide yearly,[1] of which clear cell RCC (cCRCC) is the most common, representing approximately $75 \%$ of all adult renal malignancies. The survival rate of ccRCC patients has tremendously improved owing to the advancements in early detection techniques. Patients with localised tumors were treated with nephrectomy. However, regional or distant metastases occur in one-third of patients with ccRCC. Therefore, the development of new systemic therapies, including molecular targeted therapy and immunotherapy, is required for metastatic ccRCC.

The inactivation of von Hippel Lindau (VHL) is frequently observed in ccRCC,[2-5] resulting in impaired ubiquitination and the accumulation of hypoxia-inducible transcription factor (HIF), which induces the expression of various hypoxia-related genes involved in angiogenesis.[6] Several signaling pathways have also been reported to be activated in ccRCC cells.[7] Based on these findings, molecular target 
therapy directed toward vascular endothelial growth factor (VEGF) and mammalian target of rapamycin (mTOR) have developed over the past two decades.[8-12] However, their therapeutic effects remain limited.[13,14]

Other mechanisms have been revealed to play important roles in the ccRCC progression and are thus expected to be potential targets for treatment. For instance, epigenetic modifications are important not only for carcinogenesis but also for metastasis of ccRCC.[15] Particularly, somatic mutations of genes related to histone modification have been confirmed in cCRCC. Alterations in polybromo 1 (PBRM1), breast cancer early onset (BRCA) associated protein 1 (BAP1), SET domain containing 2 (SETD2), and enhancer zeste 2 polycomb repressive complex 2 subunit ( $E Z H 2)$ are closely associated with clinical outcomes in ccRCC patients.[15-19] These chromatin regulators affect a large number of gene transcriptions, which promote the heterogeneity and evolution of ccRCC cells.[20, 21] We have also previously demonstrated that inflammation-related signalling is constitutively activated in advanced ccRCC through the formation of a super-enhancer.[22]

In addition to histone modification, cytosine residue of DNA methylation is implicated in the ccRCC progression.[23] de novo DNA methylation is induced mainly at 5'-C-phosphate-G-3' (CpG) dinucleotides by DNA methyltransferase (DNMT)3A and DNMT3B, and this methylation process is maintained by DNMT1.[24] The methylation of DNA cytosine bases leads to the inaccessibility of transcription factors to DNA regulatory elements, which in turn silences the transcription of tumor-suppressor genes.[25-28] Recent studies have revealed that DNA hypermethylation is frequently observed in CcRCC and correlates with poor prognosis.[16] However, unlike in other cancers, the methylated genes responsible for cancer progression are still unclear in ccRCC. Here, we identified the target(s) for DNMTs in ccRCC cells using genome-wide analysis, and their function in the regulation of cellular survival was evaluated.

\section{Methods}

\section{Cell culture and reagents}

Human ccRCC OS-RC-2 (RIKEN Cell Bank, Ibaraki, Japan) and their derivatives were cultured in the Roswell Park Memorial Institute 1640 medium (Thermo Fisher Scientific, Waltham, MA) containing 10\% fetal bovine serum (FBS, Thermo Fisher Scientific). Human normal proximal tubule cells HK-2 (American Type Culture Collection (ATCC), Manassas, VA) were cultured in Dulbecco's modified Eagle's medium/nutrient mixture F-12 (DMEM/F-12) medium (Thermo Fisher Scientific) containing $10 \%$ FBS. The HEK293 variant 293FT (Thermo Fisher Scientific) was cultured according to the manufacturer's protocol. Highly malignant derivatives (OS $5 \mathrm{~K}-1,-2$, and -3 cells) were established and maintained as previously described.[22] The protein kinase inhibitor staurosporine (Abcam, Cambridge, UK) and the DNMT inhibitor 5-aza-deoxycytidine (dC), (Sigma-Aldrich, St Louis, MO) were reconstituted in dimethyl sulfoxide (DMSO). The mTOR inhibitor everolimus (RAD001, Selleck Chemicals, Houston, TX) was used.

\section{Lentiviral vector construction and production}


The lentiviral vector system (provided by $\mathrm{H}$. Miyoshi, deceased, formerly Keio University) was used for specific gene overexpression and knockdown as previously described.[29] For UQCRH overexpression, the complementary DNA encoding human UQCRH was inserted into the multiple cloning site of the empty vector pENTR201. Recombination between pENTR201 and the destination vector CSII-CMV-RfA was performed using Gateway cloning technology (Thermo Fisher Scientific). The pCSII-EF-enhanced green fluorescent protein (GFP) was produced as previously described.[29] For UQCRH knockdown, short hairpin RNAs (shRNAs) targeting UQCRH were inserted into the entry vector pENTR4-H1. The target sequences for shRNA are shown in Table 1 or as reported previously.[30] Recombination between pENTR4-H1 and the destination vector pCS-RfA was performed using Gateway cloning technology. The prepared plasmids, pCAG-HIVgp, and pCMV-VSV-G-RSV-Rev, were transfected into 293FT cells using Lipofectamine 2000 (Thermo Fisher Scientific). The lentiviral vectors were collected from the culture supernatants and concentrated using the Lenti-X Concentrator (Clontech, Mountain View, CA).

\section{Quantitative RT-PCR (qRT-PCR) analysis}

qRT-PCR analysis was performed as previously described.[30] Total RNA was extracted using the ISOGEN Reagent (Nippon Gene, Toyama, Japan) or an RNeasy Mini Kit (Qiagen, Hilden, Germany). cDNA was synthesized using the PrimeScript II 1st strand cDNA synthesis kit (Takara Bio, Shiga, Japan), and the cDNA products were mixed with FastStart Universal SYBR Green Master Mix with ROX (Roche Diagnostics, Basel, Switzerland) and analyzed on StepOnePlus Real-Time PCR system (Thermo Fisher Scientific). The expression levels of human UQCRH mRNAs were normalized to that of human ACTB mRNA. The primer sequences are shown in Table 2.

\section{Immunoblotting}

Immunoblotting was performed as previously described.[31] Cells were collected, washed with phosphate-buffered saline (PBS), and lysed in RIPA buffer (1\% Triton X-100, $0.5 \%$ deoxycholate, $0.1 \%$ sodium dodecyl sulfate (SDS), $150 \mathrm{mM} \mathrm{NaCl}$, and $50 \mathrm{mM}$ Tris-HCl at pH 8.0) containing $1 \times$ cOmplete Protease Inhibitor Cocktail (Roche Diagnostics). Cytochrome release was measured using a Cytochrome $c$ Release Assay Kit (Abcam) according to the manufacturer's instructions, subjected to SDSpolyacrylamide gel electrophoresis and transferred onto polyvinyl fluoride membranes (Pall Corporation, East Hills, NY) blocked with $5 \%$ skim milk containing tris-buffered saline with Tween20 (Sigma-Aldrich) (TBST). The membranes were incubated with primary antibodies and the appropriate secondary antibodies (Table 3) diluted in TBST, Can Get Signal 1 (Toyobo, Osaka, Japan), or Can Get Signal 2 (Toyobo). Chemiluminescence images were captured using an ImageQuant LAS 4000 device (Fuji Film, Tokyo, Japan).

\section{Immunohistochemistry and TdT-mediated dUTP nick end labeling (TUNEL) assay}

For immunostaining, mouse tissues were fixed with Mildform (Wako Pure Chemical, Tokyo, Japan), whereas for human renal tumor tissues, a pre-fixed human tissue array was purchased (KD2082a, US Biomax Inc., Rockville, MD). After paraffinisation, the tissues were sectioned and deparaffinized using 
xylene and ethanol, followed by antigen retrieval using Universal HIER Antigen Retrieval Reagent (Abcam). Samples were subjected to hematoxylin and eosin staining or immunostaining. After blocking with Block ACE (Bio-Rad, Hercules, CA), the samples were incubated with primary antibodies and the appropriate secondary antibodies (Table 4) and stained using a Dako Liquid DAB+ Substrate Chromogen System (Agilent Technologies, CA) and Mayor's hematoxylin. Images were captured using an AX80 microscope (Olympus, Tokyo, Japan).

To detect apoptosis, In Situ Cell Death Detection Kit (TMR red; Roche Diagnostics) and DAPI Fuloromount-G (Southern Biotech, Birmingham, AL) were used as previously described.[32] Fluorescent images were captured using a BZ-X710 microscope (KEYENCE, Osaka, Japan).

\section{Immunocytochemistry}

Cells were plated on Matsunami Micro Cover Glass (Matsunami, Osaka, Japan), fixed with $4 \%$ paraformaldehyde solution, and permeabilized with $0.1 \%$ Triton-X-100 containing Tween 20 . Then, they were stained with anti-cytochrome $c$ antibody (12963; 1:300, Cell Signaling Technology, Danvers, MA) and visualized using anti-mouse IgG H\&L secondary antibodies (Alexa Fluor 488; Invitrogen) in Blocking One reagent (Nacalai Tesque, Kyoto, Japan). The nuclei were stained with DAPI Fuloromount-G.

\section{Mouse renal orthotopic tumor models}

All experiments were approved by the Animal Ethics Committee of the University of Tokyo. Mouse renal orthotopic tumor models were generated as previously described.[29] Briefly, BALB/c-nu/nu male mice (5weeks-old) were purchased from Sankyo Labo Service Corporation (Tokyo, Japan). ccRCC cells (1.0 x $10^{5}$ ) expressing Luc2 and mCherry were inoculated into the subrenal capsule of mice. For in vivo bioluminescence imaging, D-luciferin potassium salt (200 mg/kg; Promega, Madison, WI) was diluted in PBS and injected into mice intra-peritoneally. For ex vivo bioluminescence imaging, the harvested kidneys and lungs were reacted with D-luciferin potassium solution for $10 \mathrm{~min}$, and images were captured using Night OWL LB981 (Berthold Technologies, Bad Wildbad, Germany). Quantitative analysis was conducted using the IndiGO software (Berthold Technologies). Everolimus was reconstituted in saline solution (Otsuka, Tokyo, Japan) containing 5\% Tween20 and 30\% propylene glycol (Sigma-Aldrich) and administered to mice $(2.5 \mathrm{mg} / \mathrm{kg})$ thrice weekly.

\section{Cell proliferation assay}

Cell Counting Kit-8 (Dojindo Laboratories, Kumamoto, Japan) was used according to the manufacturer's protocol.

\section{Colony formation assay}

The colony formation assay was performed as previously described.[33]

\section{Flow cytometry analysis}


Flow cytometry analysis was performed as previously described.[22] Briefly, the cells were collected, washed with Annexin $\mathrm{V}$ binding buffer (Thermo Fisher Scientific), and reacted with FITC-conjugated Annexin V (Thermo Fisher Scientific) at room temperature for 10 min. Apoptotic cells were detected using a Gallios flow cytometer (Beckman Coulter, Brea, CA).

\section{Bisulfite-sequencing analysis}

Genomic DNA extraction and bisulfite conversion were performed as described previously.[22] Briefly, genomic DNA was purified using a Gentra Puregene Cell Kit (Qiagen). Bisulfite conversion was performed using an EpiTect Bisulfite Kit (Qiagen). Bisulfited DNA was amplified with Takara Epi-Taq HS (Takara Bio) using a specific primer for the human UQCRHCpG island shore. The primer sequences are listed in Table 5. After ligation with the pCR4-TOPO vector using the TOPO TA Cloning Kit (Thermo Fisher Scientific), products were transformed into DH5a and sequenced.

\section{Methylation array}

Genomic DNA extraction was performed as described previously.[22] Five hundred ng of genomic DNAs were quantified by Qubit Fluorometer (Life Technologies, Carlsbad, CA) and bisulfite-converted using an EZ DNA Methylation Kit (Zymo Research, Irvine, CA). Methylation array was conducted using the Infinium Human MethylationEPIC BeadChip Kit (Illumina, San Diego, CA) according to the manufacturer's protocol. The raw signal intensity for methylated and unmethylated DNA was measured using a BeadArray Scanner (Illumina). After color-bias correction, background subtraction of the signal intensities, and interarray normalization on Genome Studio (Illumina), the raw methylation value ( $\beta$-value) for each $\mathrm{CpG}$ was defined as $M /(M+U+100)$, where $M$ and $U$ were the intensities of methylated and unmethylated probes, respectively. CpG loci located-0-500 bp upstream of transcript start sites (TSS) were used for the analysis of methylation status in promoters.

\section{Public database}

Data for gene expression and DNA methylation were obtained from public databases, i.e., The Cancer Genome Atlas (TCGA) program, Gene Expression Omnibus (GSE131137, GSE53757, and GSE83820) of the National Center for Biotechnology Information (NCBI), and Cancer Cell Line Encyclopedia (CCLE) of the Broad Institute.

\section{Statistical analysis}

Graph generation and statistical analysis were performed using Excel (Microsoft, Redmond, WA), JMP Pro 14.2, R (v4.0.2), and Python 3. No method was used to analyse the sample sizes. Two-group comparison was analyzed using Student's $t$-test or Welch's $t$-test based on the results of the $F$-test. For multiple comparisons, one-way analysis of variance (ANOVA), Tukey's test, and Dunnett's test were used. For the Kaplan-Meier plot analysis, a log-rank test was used. 


\section{Results}

\section{Increased expression of DNMT3B contributes to renal cancer progression}

In our previous study, orthotopic transplantations were employed to establish highly malignant derivatives of human ccRCC cells (Fig. S1A). Parental OS-RC-2 (OSPa) cells were repeatedly exposed to the renal microenvironment. After five serial orthotopic transplantations, three derivatives were obtained as OS5K-1, -2 , and -3 cells. Although the proliferative ability of OS5K cells did not increase in cell culture (Figure S1B), they exhibited increased tumor formation and metastasis in 3D culture conditions or in vivo (Fig. S1C-F).

To examine the role of DNA methylation during renal cancer progression, the expression levels of DNMTs were determined using our previous RNA-sequencing (RNA-seq) data (GSE131137)[22] and immunoblotting. The expression of DNMT3B was upregulated in OS5K cells (Fig. 1A, B). The activity of DNMTs in these cells was diminished following treatment with 5-aza-dC, a DNMT inhibitor. Although the viability of OSPa and OS5K cells was decreased by 5 -aza-dC, 5-aza-dC was more potent in suppressing that of OS5K cells (Fig. 1C). When xenografted, 5-aza-dC pre-treatment attenuated the formation of primary tumor in OS5K cells, although lung metastasis was not significantly affected (Fig. 1D, E). Histological examination revealed a decrease in the number of cells with 5-methylated cytidine in the nuclei, which was accompanied by an increase in TUNEL-positive cells (Fig. 1F, G). These results suggest that DNA methylation is accelerated by DNMT3B in OS5K cells, which may account for their pro-survival phenotype.

Next, the involvement of DNA methylation in ccRCC progression was confirmed using clinical datasets. In cCRCC cases, increased expression of DNMT1 and DNMT3A was observed in a stage-dependent manner (Fig. 2A). Moreover, poor prognosis of cCRCC patients correlated with the upregulation of DNMT3A and more significantly with DNMT3B (Fig. 2B). Overall, these data suggest that the expression of DNMT3B enhances DNA methylation during CcRCC progression, which may be important in tumor formation and related to poor patient outcomes.

\section{Identification of targets for DNMT3B in renal cancer cells}

To uncover the role of DNA methylation, genome-wide screening of DNA methylated sites in ccRCC cells was performed using a methylation array (Fig. 3A). We also re-analyzed the previous RNA-seq data (GSE131137) (Fig. 3B). We confirmed that the decreased expression of several genes was correlated with DNA methylation (Fig. 3C, D). To assess the features of methylated genes, the hypermethylated genes were extracted in OS5K cells and subjected to Gene Ontology (GO) analysis. GO analysis of the biological process revealed that the genes governing cellular survival were methylated in OS5K cells, including apoptosis and necrosis (Fig. 3E). 
Next, among these methylated genes in OS5K cells, we identified those that are important for renal cancer progression. Ubiquinol cytochrome $c$ reductase hinge protein $(U Q C R H)$, one of the components of the mitochondrial complex III, was extracted as a methylation target. Compared with normal proximal tubule HK-2 cells, UQCRH expression was decreased in OSPa cells and further reduced in OS5K cells (Fig. 4A, B). When all of the components of the electron transport chain in OS5K cells were examined, neither gene expression nor methylation status was altered, except for that of UQCRH (Fig, S2).

Clinical database analysis showed that decreased expression of UQCRH was confirmed in cancer cells derived from the kidney and ovary (Fig. S3A). Using the GEO data, we found that the loss of UQCRH cells was observed during the establishment of patient-derived xenograft (PDX) using ccRCC tissues (Fig. S3B). To assess the clinical significance of the decreased expression of UQCRH, its expression in renal tumor tissues was analyzed using clinical samples. Immunohistochemical analysis revealed that the expression of UQCRH in CCRCC tissues was lower than that in normal adjacent tissues, irrespective of the tumor grade (Fig. 4C, D). Moreover, UQCRH expression in other histological types of renal tumor tissues, including papillary RCC, squamous cell carcinoma, and sarcomatoid carcinoma, was also lower than that in normal adjacent tissues.

Based on these observations, we focused on the role of UQCRH in ccRCC cells in subsequent experiments. Particularly, the methylation status of the $\mathrm{CpG}$ island of the UQCRH promoter locus was investigated. The methylation array revealed that all of the GpG loci in UQCRH were methylated in OS5K cells but not in OSPa cells (Fig. 5A). Similar results were yielded by bisulfite-sequencing analysis (Fig. 5B).

Clinical database analysis revealed that the methylation of the promoter in UQCRH was frequently observed in cancer cells from the kidney and ovary (Fig. S4A). Notably, the expression of UQCRH was inversely correlated with that of DNMT3B in CCRCC (Fig. S4B).

To directly examine the regulation of UQCRH by DNA methylation, OS5K cells were treated with the DNA methyltransferase inhibitor 5-aza-dC. The expression of UQCRH was restored at both the mRNA and protein levels (Fig. 5C, D). Taken together, these data suggest that the DNMT3B-mediated decrease in UQCRH may contribute to renal cancer progression.

\section{Renal cancer cells acquire apoptosis resistance through the decrease of UQCRH}

The role of UQCRH expression in apoptosis induction was further investigated. In OSPa cells, the translocation of cytochrome $c$ from the mitochondria to the cytosol, an indicator of the initiation of the apoptotic process, was observed following staurosporine treatment (Fig. 6A, B). Subsequently, the cleavage of poly (ADP-ribose) polymerase (PARP), which indicates caspase activation (Fig. S5A), was observed. However, these apoptotic processes were suppressed in OS5K cells even after treatment with staurosporine. 
Next, the involvement of UQCRH in apoptosis induction was examined using overexpression and knockdown experiments. The expression of UQCRH in OS5K-3 cells was recovered using lentiviral vectors (OS5K-UQCRH cells) (Fig. S5B, C). Cytochrome $c$ was not released into the cytoplasm in the control OS5KGFP cells after staurosporine treatment, whereas it was efficiently released in OS5K-UQCRH cells (Fig. S5D). We also established UQCRH-silenced OSPa cells using shRNAs (OSPa-shUQCRH \#1, \#2 cells) (Fig. 6C, D). Immunoblot analysis revealed that the introduction of shUQCRHs inhibited the translocation of cytochrome $c$ and the cleavage of PARP (Fig. 6E, Fig. S5E). Although 5-aza-dC treatment decreased the viability of the control OSPa-shNTC cells, this effect was partially attenuated in OSPa-shUQCRH cells (Fig. 6F).

Then, the tumorigenic potentials of OSPa-shNTC and OSP-shUQCRH cells were compared using a mouse renal orthotopic tumor model. When xenografted, OSPa-shUQCRH cells exhibited significantly faster primary tumor formation than OSPa-shNTC cells, while lung metastasis was not different in each cell type (Fig. 6G, Fig. S5F). The number of apoptotic cells was lower in tumor tissues derived from OSPashUQCRH cells than that from OSPa-shNTC cells (Fig. 6H). These results suggest that UQCRH is essential for the induction of apoptosis and tumor suppression during renal cancer progression.

\section{DNMT inhibitor enhances sensitivity to mTOR inhibitor in ccRCC cells}

Finally, the pharmacological effects of a DNMT inhibitor on drug-induced apoptosis were examined. Treatment with the mTOR inhibitor everolimus successfully inhibited the activation of mTOR and the signaling of its downstream ribosomal protein S6 kinase $\beta-1$ (S6K1) in OSPa and OS5K cells equally (Fig. S6). Nonetheless, everolimus significantly induced apoptosis in OSPa cells, and less potently that of OS5K cells (Fig. 7A). When OS5K cells were pre-treated with 5-aza-dC, the everolimus-induced apoptosis was enhanced (Fig. 7B).

The sensitizing effect of 5-aza-dC was further examined in vivo. Mice were orthotopically inoculated with OS5K cells, in which the reduced expression of UQCRH was recovered by treatment with 5-aza-dC in advance as shown in Fig. 5. Although 5-aza-dC pre-treatment did not enhance the effect of everolimus on lung metastasis (at least in the concentration we tested), it significantly augmented the therapeutic effect on primary tumor formation (Fig. 7C, D). These results suggest that DNMT inhibitor potently enhances the sensitivity of ccRCC cells to mTOR inhibitors through the recovered expression of UQCRH.

\section{Discussion}

Epigenetic alterations are widely recognized in various human cancer cells.[34-39] DNA hypermethylation of promoters is induced by the inactivation of DNA demethylases or the overexpression of DNA methyltransferases.[40, 41] Additionally, mutations in genes encoding metabolism-related enzymes enhance DNA methylation status. Here, we found the up-regulation of DNMT3B in the highly malignant derivatives of ccRCC cells obtained from serial orthotopic inoculations (Fig. 1A, B). Although we have previously established highly metastatic derivatives of pancreatic cancer cells using a similar 
strategy,[42] the expression level of DNMTs was not increased in highly metastatic derivates of pancreatic cancer cells (GSE107960), suggesting that the interactions between cancer cells and the renal microenvironment may be crucial for the upregulation of DNMT3B. Several regulatory mechanisms are speculated to be involved in the increased expression of DNMT3 in cancer cells. For instance, interleukin (IL)-6 induced the expression of DNMT3B in a STAT3-dependent manner, resulting in the accelerated proliferation of ccRCC cells or oral squamous cell carcinoma cells. $[43,44]$ Similarly, here, RNA-seq results revealed the enrichment of hallmarks of IL-6-janus kinase (JAK) - signal transducer and the activator of transcription (STAT) 3 signaling in OS5Ks (unpublished data). The regulation of DNMTs by microRNAs (miRNAs) has also been described in various cancer cells.[45] Although miR4465 and miR-29c-3p may regulate the expression of DNMT3B,[46, 47] this was not evident in OS5K cells (unpublished data).

In addition, recent studies have identified many targets for DNMT3B, including tumor-suppressor genes in cancer cells.[48] Colorectal cancer, one of the most common DNA methylated cancers, exhibits decreased expression of the cyclin-dependent kinase inhibitor 2A (CDKN2A) and Ras association (RalGDS/AF-6) domain family member 1 (RASSF1A).[49, 50] Cadherin 1 (CDH1), CDKN2A, the runt-related transcription factor 3 (RUNX3), BRCA1, and RASSF1A are reported to be suppressed by DNMT3B-dependent DNA hypermethylation in gastric or breast cancers.[51, 52] In the present study, we conducted genome-wide screening of methylation targets in ccRCC cells using RNA-seq analysis and methylation array. UQCRH was chosen as a candidate gene whose expression was epigenetically decreased in highly malignant derivatives in ccRCC cells. Decreased expression of UQCRH was also validated using a tissue array of clinical ccRCC cases (Fig. 4). These observations are in accordance with the results obtained using clinical ccRCC samples in other cohorts,[16] indicating that the downregulation of UQCRH is common in cCRCC. The expression of UQCRH is similarly decreased in several types of cancers. [53] Among them, we found that decreased expression of UQCRH in clear cell carcinoma of ovarian cancer (Fig. S3A). The histological phenotype of clear cell carcinoma is likely to be associated with the downregulation of UQCRH, irrespective of the origin of cancers.

UQCRH is highly conserved in many species and serves as a component of the mitochondrial complex III. It is closely associated with the function of cytochrome $c$ and cytochrome $c 1$.[54-56] Although UQCRH is also involved in electron transport and the maturation of cytochrome $c 1,[57-59]$ its detailed role in the maintenance of the proton gradient in the inner mitochondrial membrane remains unknown. The survival of blood cells was enhanced by the introduction of UQCRH.[60] During the preparation of this manuscript, Luo and colleagues reported a decrease in UQCRH and its functions in the Warburg effect in renal cell carcinoma.[61] Given these reports, we speculate that UQCRH contributes to the induction of apoptosis. In the absence of an apoptotic stimuli, cytochrome $c$ is anchored to the inner mitochondrial membrane by binding to cardiolipin. This binding is attenuated when the inner mitochondrial membrane is oxidized by the accumulation of reactive oxygen species (ROS), resulting in the release of cytochrome c.[62-64] Since UQCRH regulates the production of ROS,[58] we hypothesized that UQCRH may be essential for the induction of apoptosis in cancer cells. Accordingly, we showed that the silencing of UQCRH attenuated the translocation of cytochrome $c$ and the apoptosis in ccRCC cells treated with staurosporine (Fig. 6 and 
Fig, S5). These findings suggest that UQCRH may serve as a potential tumor suppressor in ccRCC through the regulation of apoptosis.

DNMT inhibitors, namely 5-azacytidine and 5-aza-2'-dC, were initially considered for cancer treatment. However, due to their toxicity, only a low dose is recommended. A clinical trial of DNMT inhibitors in combination with other anti-cancer drugs, such as IL-2, interferon (IFN)- $a$, and bevacizumab, for renal cancer treatment has been conducted.[23] Here, our results demonstrated that the silencing of UQCRH enhances the drug-induced apoptosis in ccRCC cells (Fig. 6) and that the 5-aza-dC treatment sensitizes the anti-tumor effect of mTOR inhibitor both in vivo and in vitro (Fig. 7). Our results contribute to the further understanding of the molecular mechanisms involved in the drug resistance of ccRCC cells and provide an important insight into therapeutic options for ccRCC.

\section{Conclusions}

Here, we report the tumor progressive role of DNMT3B in ccRCC. Our results suggest that DNA methylation causes the decreased expression of the potential tumor-suppressor gene UQCRH, which is essential for the completion of the apoptotic process in ccRCC cells. In our preclinical study, DNA demethylation induced by 5 -aza-dC enhanced the tumor-suppressive ability of everolimus. These findings confirms the clinical potential of DNMT inhibitors for ccRCC treatment.

\section{Abbreviations}

CCRCC

clear cell renal cell carcinoma

DNMT

DNA methyltransferase

UQCRH

ubiquinol cytochrome $c$ reductase hinge protein

mTOR

mammalian target of rapamycin

shRNA

short hairpin RNA

qRT-PCR

quantitative RT-PCR

TUNEL

TdT-mediated dUTP nick end labeling

GFP

enhanced green fluorescent protein

RNA-seq

RNA-sequencing 


\section{Declarations}

\section{Ethics approval and consent to participate}

All animal experiments were approved by the Animal Ethics Committee of the University of Tokyo. Human tumor tissue array was obtained commercially.

\section{Consent for publication}

Not applicable.

\section{Availability of data and materials}

Raw and processed RNA-seq data are available at Gene Expression Omnibus (GEO). The additional data that support the findings of this study are available from the corresponding author upon reasonable request.

\section{Competing interests}

We declare no potential conflict of interest.

\section{Funding}

This work was supported by a KAKENHI Grant-in-Aid for Scientific Research on Innovative Area on Integrated Analysis and Regulation of Cellular Diversity from the Ministry of Education, Culture, Sports, Science and Technology (MEXT) of Japan (17H06326 to K. Miyazono and S.E.); a KAKENHI Grant-in-Aid for Scientific Research (C) from the Japan Society for the Promotion of Science (JSPS) (19K07684 to S.E.); the Princess Takamatsu Cancer Research Fund (to S.E.). This work was also in part supported by a grant for Endowed Department (Department of Medical Genomics) from Eisai Co., Ltd.

\section{Authors' contributions}

K. Miyakuni and S.E. conceived the study. K. Miyakuni and J.N. performed most of the experiments. D.K., G.N., and H.A. assisted in the methylation array. K.Miyazono and S.E. supervised the project and wrote the manuscript. All authors discussed the results and commented on the manuscript.

\section{Acknowledgements}

We thank Y. Morishita, K. Takahashi (Graduate School of Medicine, The University of Tokyo), and Hiroko Meguro (Research Center for Advanced Science and Technology, The University of Tokyo) for their technical assistance. We also thank H. Miyoshi (deceased, formerly Keio University) for providing the lentiviral vectors.

\section{References}


1 Rini BI, Campbell SC, Escudier B. Renal cell carcinoma. Lancet. 2009;373:1119-1132.

2 Gnarra JR, Tory K, Weng Y, Schmidt L, Wei MH, Li H et al. Mutations of the VHL tumour suppressor gene in renal carcinoma. Nat Genet. 1994;7:85-90.

3 Gallou C, Joly D, Mejean A, Staroz F, Martin N, Tarlet G et al. Mutations of the VHL gene in sporadic renal cell carcinoma: Definition of a risk factor for VHL patients to develop an RCC. Human Mutation. 1999;13:464-475.

4 Schraml P, Struckmann K, Hatz F, Sonnet S, Kully C, Gasser T et al. VHL mutations and their correlation with tumour cell proliferation, microvessel density, and patient prognosis in clear cell renal cell carcinoma. Journal of Pathology. 2002;196:186-193.

5 Herman JG, Latif F, Weng Y, Lerman MI, Zbar B, Liu S et al. Silencing of the VHL tumor-suppressor gene by DNA methylation in renal carcinoma. Proc Natl Acad Sci U S A. 1994;91:9700-9704.

6 Kaelin WG. Von Hippel-Lindau disease. Annu Rev Pathol. 2007;2:145-173.

7 Brugarolas J. Renal-cell carcinoma - Molecular pathways and therapies. N Engl J Med. 2007;356:185187.

8 Choueiri TK, Motzer RJ. Systemic Therapy for Metastatic Renal-Cell Carcinoma. N Engl J Med. 2017;376:354-366.

9 Motzer RJ, Hutson TE, Tomczak P, Michaelson MD, Bukowski RM, Rixe 0 et al. Sunitinib versus interferon alfa in metastatic renal-cell carcinoma. N Engl J Med. 2007;356:115-124.

10 Hudes G, Carducci M, Tomczak P, Dutcher J, Figlin R, Kapoor A et al. Temsirolimus, interferon alfa, or both for advanced renal-cell carcinoma. N Engl J Med. 2007;356:2271-2281.

11 Escudier B, Eisen T, Stadler WM, Szczylik C, Oudard S, Siebels M et al. Sorafenib in advanced clear-cell renal-cell carcinoma. N Engl J Med. 2007;356:125-134.

12 Dizman N, Philip EJ, Pal SK. Genomic profiling in renal cell carcinoma. Nat Rev Nephrol. 2020;16:435451.

13 Kim WY, Kaelin WG. Role of VHL gene mutation in human cancer. J Clin Oncol. 2004;22:4991-5004.

14 Ricketts CJ, De Cubas AA, Fan H, Smith CC, Lang M, Reznik E et al. The cancer genome atlas comprehensive molecular characterization of renal cell carcinoma. Cell Rep. 2018;23:313-326.e315.

15 de Cubas AA, Rathmell WK. Epigenetic modifiers: activities in renal cell carcinoma. Nat Rev Urol. 2018;15:599-614.

16 Comprehensive molecular characterization of clear cell renal cell carcinoma. Nature. 2013;499:43-49. 
17 Dalgliesh GL, Furge K, Greenman C, Chen L, Bignell G, Butler A et al. Systematic sequencing of renal carcinoma reveals inactivation of histone modifying genes. Nature. 2010;463:360-363.

18 Guo G, Gui Y, Gao S, Tang A, Hu X, Huang Y et al. Frequent mutations of genes encoding ubiquitinmediated proteolysis pathway components in clear cell renal cell carcinoma. Nat Genet. 2011;44:17-19.

19 Varela I, Tarpey P, Raine K, Huang D, Ong CK, Stephens P et al. Exome sequencing identifies frequent mutation of the SWI/SNF complex gene PBRM1 in renal carcinoma. Nature. 2011;469:539-542.

20 Kanu N, Grönroos E, Martinez P, Burrell RA, Yi Goh X, Bartkova J et al. SETD2 loss-of-function promotes renal cancer branched evolution through replication stress and impaired DNA repair. Oncogene. 2015;34:5699-5708.

21 Gerlinger M, Rowan AJ, Horswell S, Math M, Larkin J, Endesfelder D et al. Intratumor heterogeneity and branched evolution revealed by multiregion sequencing. N Engl J Med. 2012;366:883-892.

22 Nishida J, Momoi Y, Miyakuni K, Tamura Y, Takahashi K, Koinuma D et al. Epigenetic remodelling shapes inflammatory renal cancer and neutrophil-dependent metastasis. Nat Cell Biol. 2020;22:465-475.

23 Joosten SC, Smits KM, Aarts MJ, Melotte V, Koch A, Tjan-Heijnen VC et al. Epigenetics in renal cell cancer: mechanisms and clinical applications. Nat Rev Urol. 2018;15:430-451.

24 Gao L, Emperle M, Guo Y, Grimm SA, Ren W, Adam S et al. Comprehensive structure-function characterization of DNMT3B and DNMT3A reveals distinctive de novo DNA methylation mechanisms. Nat Commun. 2020;11:3355.

25 Pfeifer GP. Defining Driver DNA Methylation Changes in Human Cancer. Int J Mol Sci. 2018;19.

26 Baubec T, Colombo DF, Wirbelauer C, Schmidt J, Burger L, Krebs AR et al. Genomic profiling of DNA methyltransferases reveals a role for DNMT3B in genic methylation. Nature. 2015;520:243-247.

27 Gagliardi M, Strazzullo M, Matarazzo MR. DNMT3B Functions: Novel Insights From Human Disease. Front Cell Dev Biol. 2018;6:140.

28 Zhang Y, Charlton J, Karnik R, Beerman I, Smith ZD, Gu H et al. Targets and genomic constraints of ectopic Dnmt3b expression. Elife. 2018;7.

29 Taguchi L, Miyakuni K, Morishita Y, Morikawa T, Fukayama M, Miyazono K et al. c-Ski accelerates renal cancer progression by attenuating transforming growth factor $\beta$ signaling. Cancer Sci. 2019;110:2063-2074.

30 Nishida J, Miyazono K, Ehata S. Decreased TGFBR3/betaglycan expression enhances the metastatic abilities of renal cell carcinoma cells through TGF- $\beta$-dependent and -independent mechanisms. Oncogene. 2018;37:2197-2212. 
31 Murai F, Koinuma D, Shinozaki-Ushiku A, Fukayama M, Miyaozono K, Ehata S. EZH2 promotes progression of small cell lung cancer by suppressing the TGF- $\beta$-Smad-ASCL1 pathway. Cell Discov. 2015;1:15026.

32 Yokoyama Y, Watanabe T, Tamura Y, Hashizume Y, Miyazono K, Ehata S. Autocrine BMP-4 Signaling Is a Therapeutic Target in Colorectal Cancer. Cancer Res. 2017;77:4026-4038.

33 Takahashi K, Ehata S, Koinuma D, Morishita Y, Soda M, Mano H et al. Pancreatic tumor microenvironment confers highly malignant properties on pancreatic cancer cells. Oncogene. 2018;37:2757-2772.

34 Pfister SX, Ashworth A. Marked for death: targeting epigenetic changes in cancer. Nat Rev Drug Discov. 2017;16:241-263.

35 Jones PA, Laird PW. Cancer epigenetics comes of age. Nat Genet. 1999;21:163-167.

36 Baylin SB, Herman JG. DNA hypermethylation in tumorigenesis: epigenetics joins genetics. Trends Genet. 2000;16:168-174.

37 Goelz SE, Vogelstein B, Hamilton SR, Feinberg AP. Hypomethylation of DNA from benign and malignant human colon neoplasms. Science. 1985;228:187-190.

38 Baylin SB. DNA methylation and gene silencing in cancer. Nat Clin Pract Oncol. 2005;2 Suppl 1:S4-11.

39 Rhee I, Bachman KE, Park BH, Jair KW, Yen RW, Schuebel KE et al. DNMT1 and DNMT3b cooperate to silence genes in human cancer cells. Nature. 2002;416:552-556.

40 Delhommeau F, Dupont S, Della Valle V, James C, Trannoy S, Massé A et al. Mutation in TET2 in myeloid cancers. N Engl J Med. 2009;360:2289-2301.

41 Mizuno S, Chijiwa T, Okamura T, Akashi K, Fukumaki Y, Niho Y et al. Expression of DNA methyltransferases DNMT1, 3A, and 3B in normal hematopoiesis and in acute and chronic myelogenous leukemia. Blood. 2001;97:1172-1179.

42 Takahashi K, Ehata S, Miyauchi K, Morishita Y, Miyazawa K, Miyazono K. Neurotensin receptor 1 signaling promotes pancreatic cancer progression. Mol Oncol. 2021;15:151-166.

43 Chen WC, Chen MF, Lin PY. Significance of DNMT3b in oral cancer. PLoS One. 2014;9:e89956.

44 Quan Z, He Y, Luo C, Xia Y, Zhao Y, Liu N et al. Interleukin 6 induces cell proliferation of clear cell renal cell carcinoma by suppressing hepaCAM via the STAT3-dependent up-regulation of DNMT1 or DNMT3b. Cell Signal. 2017;32:48-58.

45 Karimzadeh MR, Pourdavoud P, Ehtesham N, Qadbeigi M, AsI MM, Alani B et al. Regulation of DNA methylation machinery by epi-miRNAs in human cancer: emerging new targets in cancer therapy. Cancer 
Gene Ther. 2020.

46 Roquid KAR, Alcantara KMM, Garcia RL. Identification and validation of mRNA 3'untranslated regions of DNMT3B and TET3 as novel competing endogenous RNAs of the tumor suppressor PTEN. Int J Oncol. 2020;56:544-558.

47 Wu H, Zhang W, Wu Z, Liu Y, Shi Y, Gong J et al. miR-29c-3p regulates DNMT3B and LATS1 methylation to inhibit tumor progression in hepatocellular carcinoma. Cell Death Dis. 2019;10:48.

48 Baylin SB, Jones PA. Epigenetic Determinants of Cancer. Cold Spring Harb Perspect Biol. 2016;8.

49 Petko Z, Ghiassi M, Shuber A, Gorham J, Smalley W, Washington MK et al. Aberrantly methylated CDKN2A, MGMT, and MLH1 in colon polyps and in fecal DNA from patients with colorectal polyps. Clin Cancer Res. 2005;11:1203-1209.

50 Nosho K, Shima K, Irahara N, Kure S, Baba Y, Kirkner GJ et al. DNMT3B expression might contribute to CpG island methylator phenotype in colorectal cancer. Clin Cancer Res. 2009;15:3663-3671.

51 Llorca-Cardeñosa MJ, Fleitas T, Ibarrola-Villava M, Peña-Chilet M, Mongort C, Martinez-Ciarpaglini C et al. Epigenetic changes in localized gastric cancer: the role of RUNX3 in tumor progression and the immune microenvironment. Oncotarget. 2016;7:63424-63436.

52 Krassenstein R, Sauter E, Dulaimi E, Battagli C, Ehya H, Klein-Szanto A et al. Detection of breast cancer in nipple aspirate fluid by CpG island hypermethylation. Clin Cancer Res. 2004;10:28-32.

53 Modena P, Testi MA, Facchinetti F, Mezzanzanica D, Radice MT, Pilotti S et al. UQCRH gene encoding mitochondrial Hinge protein is interrupted by a translocation in a soft-tissue sarcoma and epigenetically inactivated in some cancer cell lines. Oncogene. 2003;22:4586-4593.

54 Smith PM, Fox JL, Winge DR. Reprint of: Biogenesis of the cytochrome bc1 complex and role of assembly factors. Biochim Biophys Acta. 2012;1817:872-882.

55 Ndi M, Marin-Buera L, Salvatori R, Singh AP, Ott M. Biogenesis of the bc1 complex of the mitochondrial respiratory chain. J Mol Biol. 2018;430:3892-3905.

$56 \mathrm{Kim} \mathrm{CH}$, King TE. A mitochondrial protein essential for the formation of the cytochrome c1-c complex. Isolation, purification, and properties. J Biol Chem. 1983;258:13543-13551.

57 Nakai M, Endo T, Hase T, Tanaka Y, Trumpower BL, Ishiwatari H et al. Acidic regions of cytochrome c1 are essential for ubiquinol-cytochrome $\mathrm{c}$ reductase activity in yeast cells lacking the acidic QCR6 protein. J Biochem. 1993;114:919-925.

58 Gao F, Liu Q, Li G, Dong F, Qiu M, Lv X et al. Identification of ubiquinol cytochrome c reductase hinge (UQCRH) as a potential diagnostic biomarker for lung adenocarcinoma. Open Biol. 2016;6. 
59 Yang M, Trumpower BL. Deletion of QCR6, the gene encoding subunit six of the mitochondrial cytochrome bc1 complex, blocks maturation of cytochrome $\mathrm{c} 1$, and causes temperature-sensitive petite growth in Saccharomyces cerevisiae. J Biol Chem. 1994;269:1270-1275.

60 Okazaki M, Ishibashi Y, Asoh S, Ohta S. Overexpressed mitochondrial hinge protein, a cytochrome cbinding protein, accelerates apoptosis by enhancing the release of cytochrome $c$ from mitochondria. Biochem Biophys Res Commun. 1998;243:131-136.

61 Luo Y, Medina Bengtsson L, Wang X, Huang T, Liu G, Murphy S et al. UQCRH downregulation promotes Warburg effect in renal cell carcinoma cells. Sci Rep. 2020;10:15021.

62 Dudek J. Role of cardiolipin in mitochondrial signaling pathways. Front Cell Dev Biol. 2017;5:90.

63 Briehl MM. Oxygen in human health from life to death-An approach to teaching redox biology and signaling to graduate and medical students. Redox Biol. 2015;5:124-139.

64 Perier C, Tieu K, Guegan C, Caspersen C, Jackson-Lewis V, Carelli V et al. Complex I deficiency primes Bax-dependent neuronal apoptosis through mitochondrial oxidative damage. Proc Natl Acad Sci U S A. 2005;102:19126-19131.

\section{Tables}

Table 1. Target sequences for shRNA.

\begin{tabular}{|lll|}
\hline Target gene & shRNA No. & Sequence $\left(\mathbf{5}^{\prime}\right.$ to $\left.\mathbf{3}^{\prime}\right)$ \\
\hline$U Q C R H$ & $\# 1$ & CGAGCAGTTGGAGAAATGT \\
\hline$U Q C R H$ & $\# 2$ & GCATCAGAATATTTCCTTA \\
\hline
\end{tabular}

Table 2. Prime sequences for qRT-PCR analysis.

\begin{tabular}{|lll|}
\hline Gene & Orientation & Sequence $\left(\mathbf{5}^{\prime}\right.$ to $\mathbf{3}^{\prime}$ ) \\
\hline ACTB & Forward & CCTGGCACCCAGCACAAT \\
\cline { 2 - 3 } & Reverse & GCCGATCCACACGGAGTACT \\
\hline UQCRH & Forward & GAGCTCTGTGATGAGCGTGT \\
\cline { 2 - 3 } & Reverse & CCCTCGCATGCAAGAAGTCA \\
\hline
\end{tabular}

Table 3. Antibodies for immunoblotting. 


\begin{tabular}{|c|c|c|c|c|}
\hline First reaction antibody & $\begin{array}{l}\text { Clone } \\
\text { number }\end{array}$ & $\begin{array}{l}\text { Catalog } \\
\text { number }\end{array}$ & Company & Dilution \\
\hline Anti-UQCRH & R40827 & HPA042574 & Sigma-Aldrich & $1 / 1000$ \\
\hline Anti-DNMT3B & 1 & 67259 & $\begin{array}{l}\text { Cell Signaling } \\
\text { Technology }\end{array}$ & $1 / 1000$ \\
\hline Anti-b-actin & 127M4857U & A5441 & Sigma-Aldrich & $1 / 1000$ \\
\hline Anti-cleaved-PARP & 15 & 9541 & $\begin{array}{l}\text { Cell Signaling } \\
\text { Technology }\end{array}$ & $1 / 1000$ \\
\hline $\begin{array}{l}\text { Anti-phospho-p70 S6 } \\
\text { kinase }\end{array}$ & 11 & 9234 & $\begin{array}{l}\text { Cell Signaling } \\
\text { Technology }\end{array}$ & $1 / 1000$ \\
\hline Anti-phospho-mTOR & 21 & 2971 & $\begin{array}{l}\text { Cell Signaling } \\
\text { Technology }\end{array}$ & $1 / 1000$ \\
\hline Anti-mTOR & 14 & 2983 & $\begin{array}{l}\text { Cell Signaling } \\
\text { Technology }\end{array}$ & $1 / 1000$ \\
\hline Anti-p70 S6 kinase & 20 & 9202 & $\begin{array}{l}\text { Cell Signaling } \\
\text { Technology }\end{array}$ & $1 / 1000$ \\
\hline
\end{tabular}

\begin{tabular}{|llll|}
\hline Second reaction antibody & Catalog number & Company & Dilution \\
\hline Anti-rabbit IgG, HRP-linked antibody & 7074 & Cell Signaling Technology & $1 / 10000$ \\
\hline Anti-mouse IgG, HRP-linked antibody & 7076 & Cell Signaling Technology & $1 / 10000$ \\
\hline
\end{tabular}

Table 4. Antibodies for immunohistochemistry.

\begin{tabular}{|lllll|}
\hline First reaction antibody & Clone number & Catalog number & Company & Dilution \\
\hline Anti-UQCRH & R40827 & HPA042574 & Sigma-Aldrich & $1 / 100$ \\
\hline Anti-5-methylcytosine & GR300572-16 & ab10805 & Abcam & $1 / 400$ \\
\hline
\end{tabular}

\begin{tabular}{|lll|}
\hline Second reaction antibody & Catalog number & Company \\
\hline Anti-rabbit lgG, HRP-linked antibody & K4003 & Dako \\
\hline Anti-mouse IgG, HRP-linked antibody & K4007 & Dako \\
\hline
\end{tabular}


Table 5. Primer sequences for bisulfite-sequencing analysis.

\begin{tabular}{|lll|}
\hline Target gene & Orientation & Sequence $\left(\mathbf{5}^{\prime}\right.$ to $\left.\mathbf{3}^{\prime}\right)$ \\
\hline UQCRH & Forward & AAGGGGATGTTTTTTAGTAAAGT \\
\cline { 2 - 3 } & Reverse & CCTAACCCAATTCAAAATCAAAA \\
\hline
\end{tabular}

Figures 

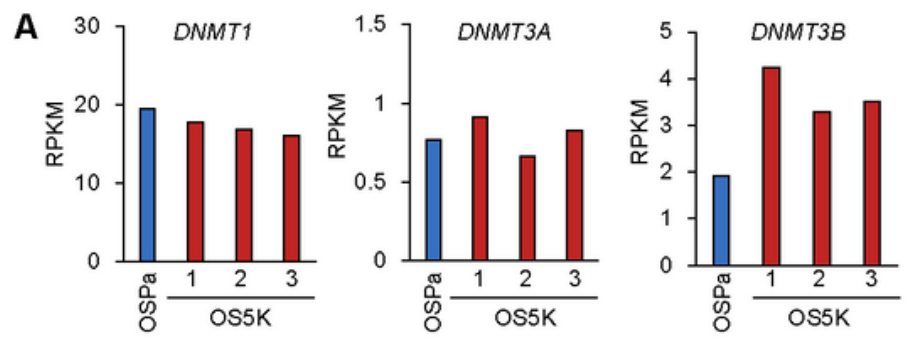
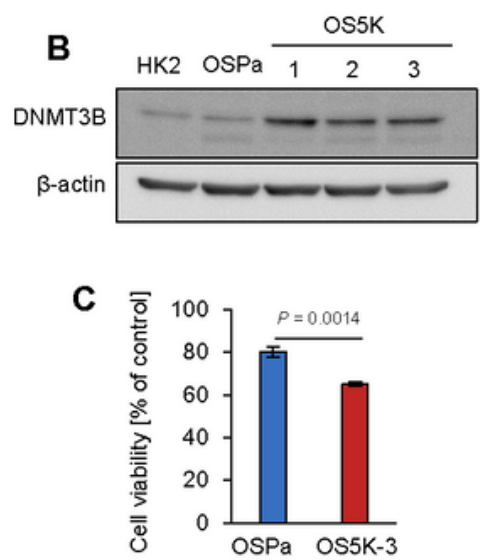

D

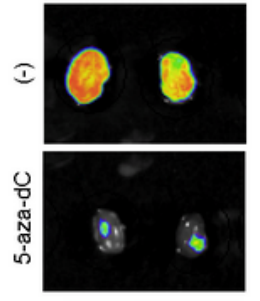

$1.0 \times 10^{5}[\mathrm{ph} / \mathrm{s}] 2.0 \times 10^{6}$

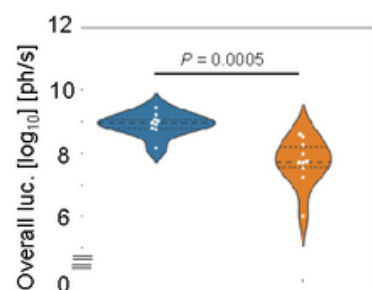

(-) 5-aza-dC OS5K-3

E

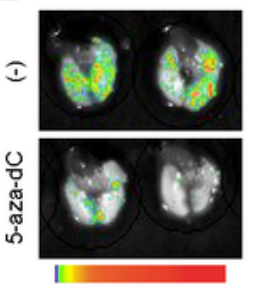

$1.0 \times 10^{1} \quad[\mathrm{ph} / \mathrm{s}] 2.0 \times 10^{3}$

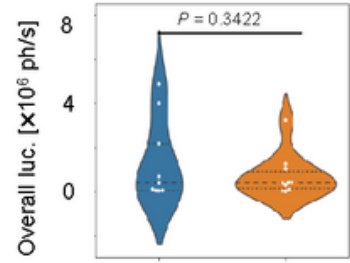

(-) 5-aza-dC

$\mathbf{F}$

$(-)$

5-aza-dC
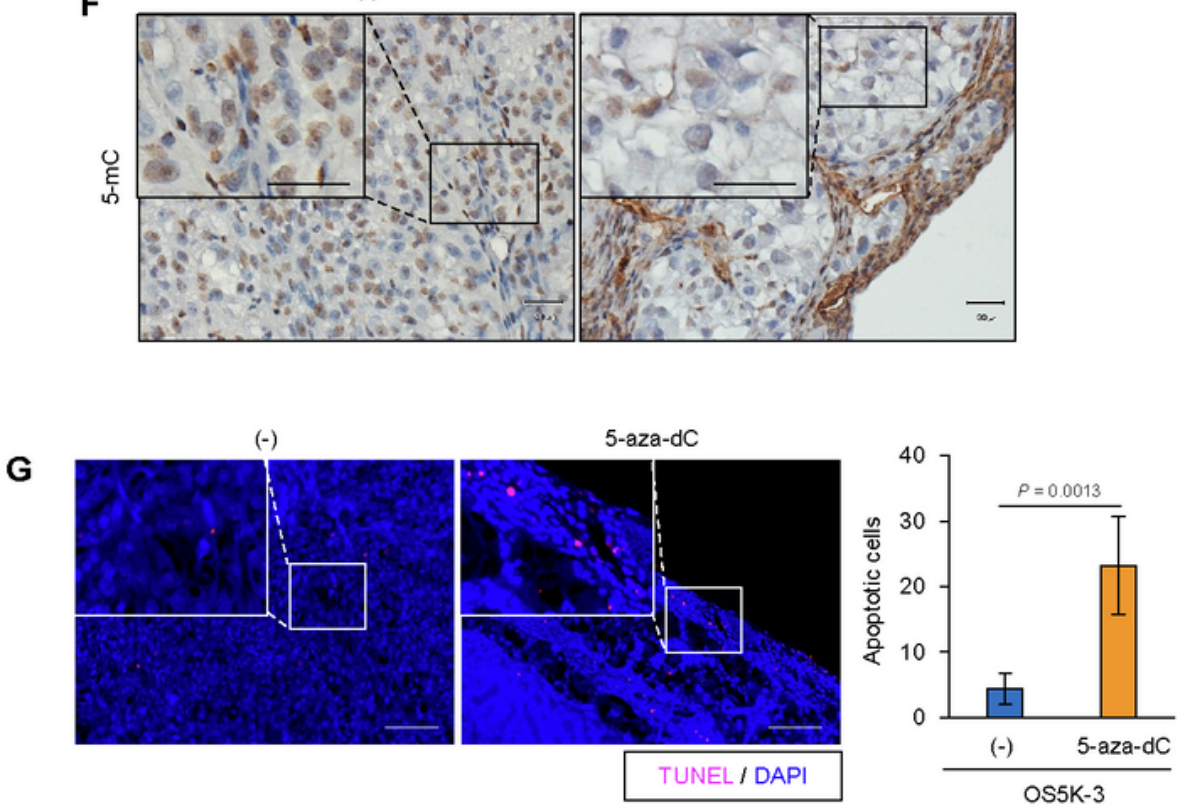

\section{Figure 1}

Inhibition of DNMTs induces apoptosis of highly malignant ccRCC cells. A. Expression of DNMTs in OSRC-2 derivatives. DNMT mRNAs were re-analyzed by RNA-seq analysis (GSE131137). ( $n=1$ sample). B. Immunoblotting of DNMT3B and $\beta$-actin expression in OS-RC-2 derivatives. C. Cell proliferation assay of OS-RC-2 derivatives. The cells were treated with 5-aza-dC $(1 \mu \mathrm{M})$ for 4 days. The percentages against untreated control of each cell are indicated. The bars represent the mean \pm SD (two-sided Student's t-test; 
$\mathrm{n}=3$ each). D, E. Ex vivo bioluminescence imaging of primary (D) and metastatic lung (E) tumors (left) and quantification (right). 0S5K-3 cells were pre-treated with or without 5 -aza-dC $(0.1 \mu \mathrm{M})$ for $72 \mathrm{~h}$ and inoculated orthotopically in mice. Mice-bearing OS-RC-2 derivatives were analyzed 16 days after orthotopic inoculation. The bars represent the mean and 1st and 3rd quartiles (D: two-sided Welch's t-test; E: two-sided Student's t-test; $n=9$, - mice; $n=10,5$-aza-dC mice). F. Immunohistochemical staining of the primary tumor tissues in $\mathrm{D}$ with 5-methylcytosine $(\mathrm{mC}) .5$-mC staining in the boxed region is shown at high magnification. Scale bars, $30 \mu \mathrm{m}$. G. TUNEL assay of the primary tumor tissues in D. Representative images (left) and the number of apoptotic cells in the independent fields (right) are shown. The nucleus was stained with DAPI. Scale bars, $100 \mu \mathrm{m}$. The bars represent the mean \pm SD (two-sided Student's t-test; $\mathrm{n}=5$ each).

A

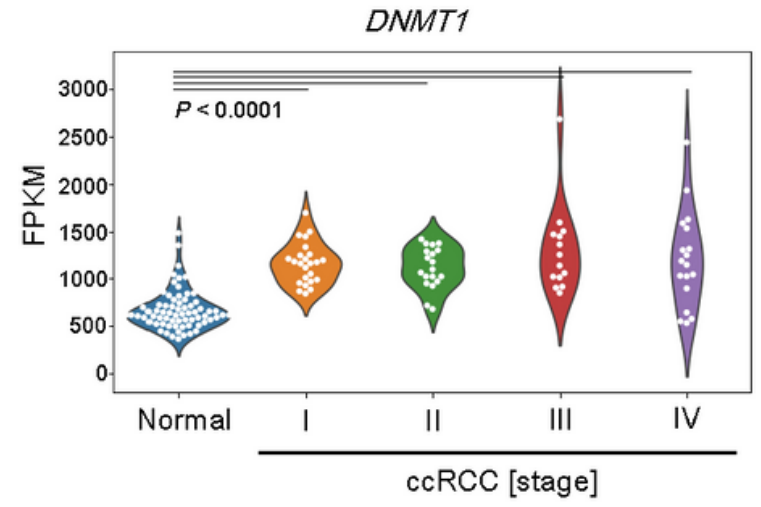

DNMT3A

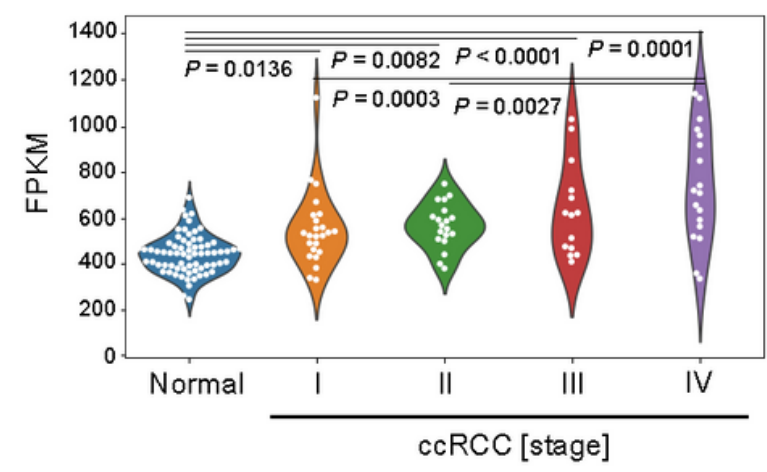

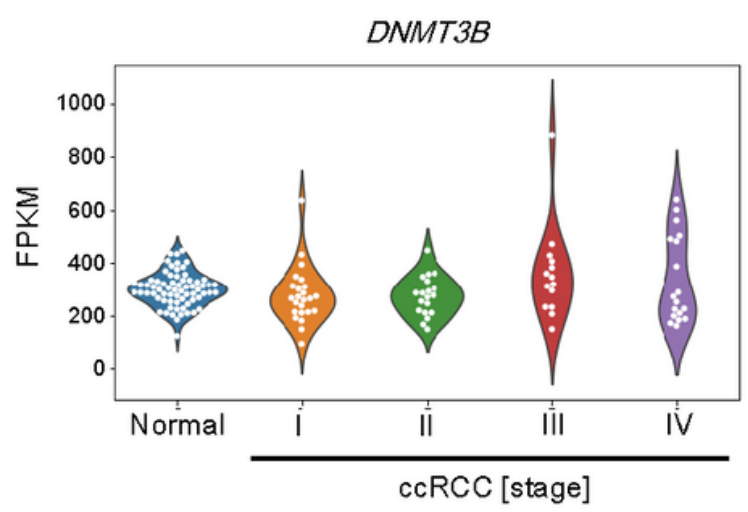

B

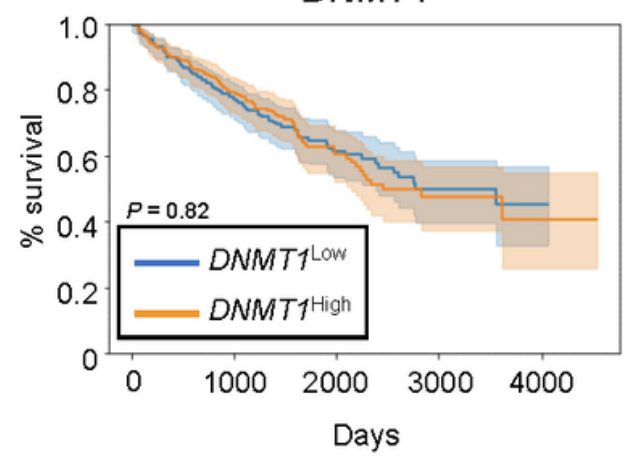

DNMT3A

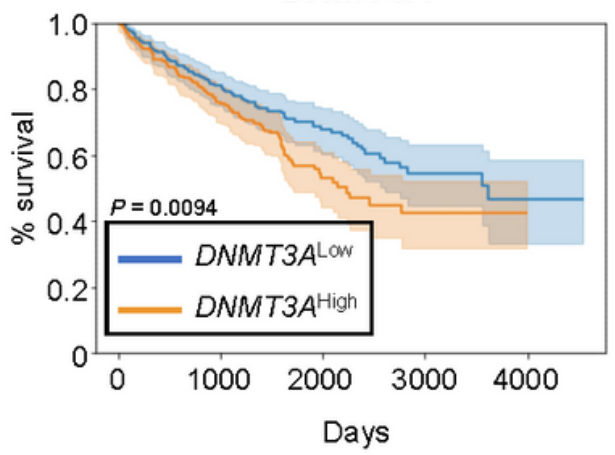

DNMT3B

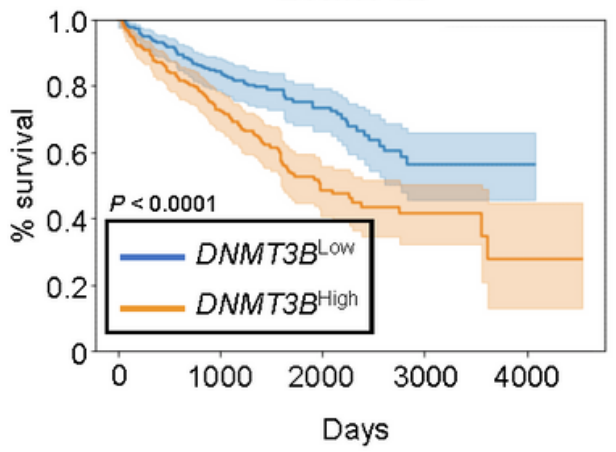




\section{Figure 2}

DNMT3B serves as a prognostic marker for renal cancer. A. Analysis of DNMT expression in ccRCC using the NCBI Gene Expression Omnibus (GEO) database (GSE53757) analyzed using One-way ANOVA and Tukey's test ( $n=69$, normal; $n=24$, ccRCC stage I; $n=19$, ccRCC stage II; $n=14$ ccRCC stage III; and $n=$ 18, ccRCC stage IV). B. Correlation between DNMT expression and the overall survival of ccRCC patients. TCGA database (KIRC) was analyzed and divided into high and low groups of each gene, respectively.

Kaplan-Meier plots show the results of applying two prognostic signatures (Log-rank test; $n=264$ each). Orange lines indicate high groups, whereas blue lines indicate low groups. 
A

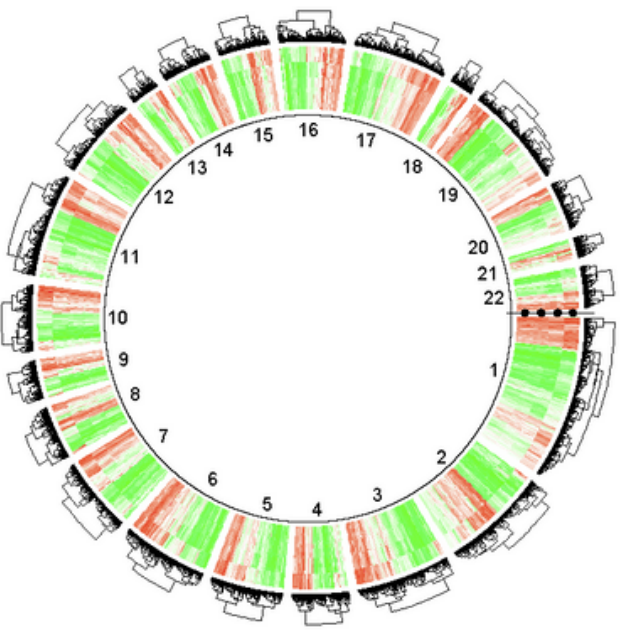

C

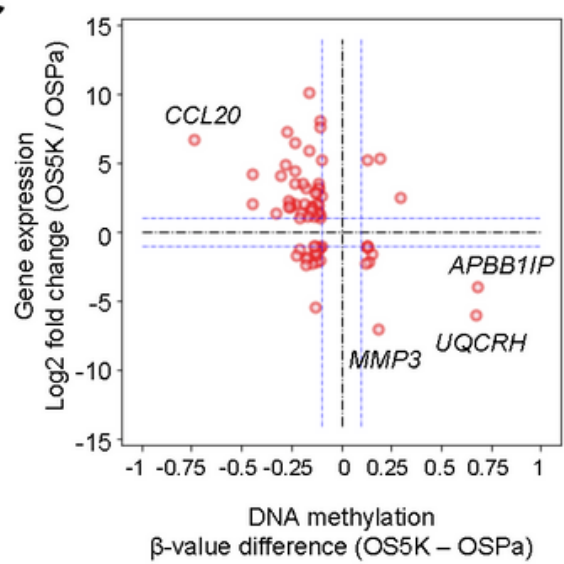

E

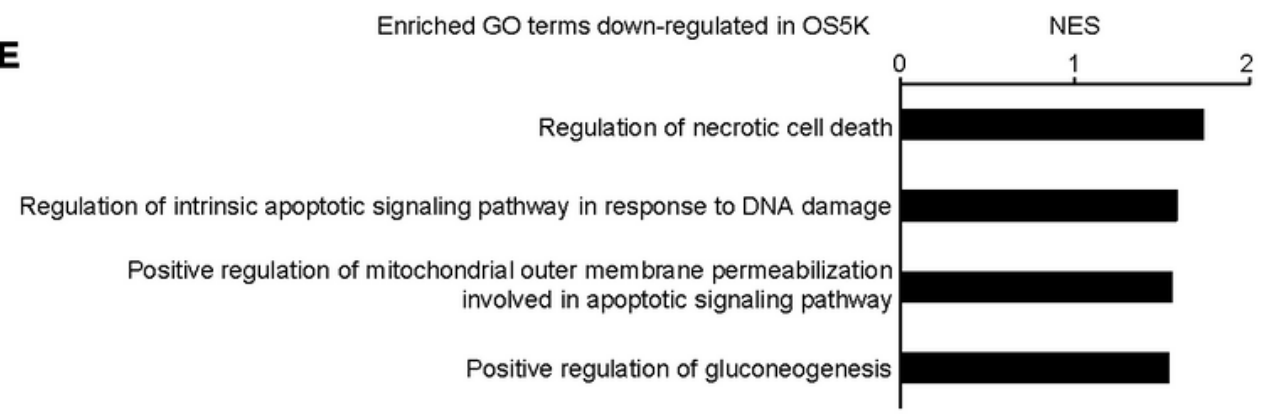

B

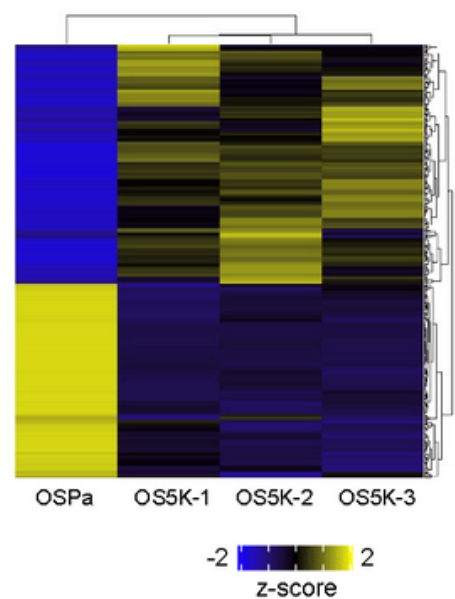

D
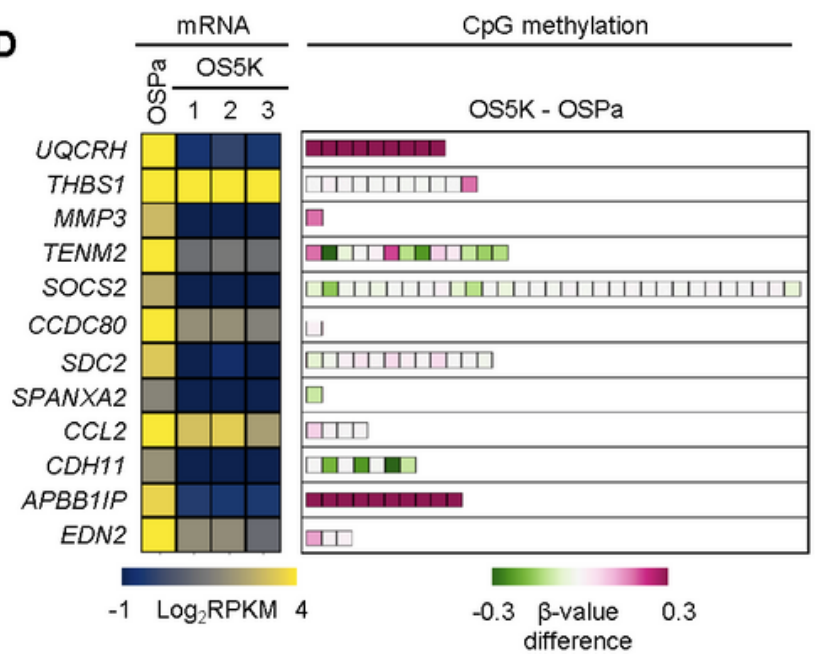

\section{Figure 3}

Integrative analysis of ccRCC derivatives using gene expression and DNA methylation in OS-RC-2 derivatives. A. Methylation status as determined by methylation array. Heatmap shows the $\beta$-value of the CpG loci in the promoter DNA in each cell. Clustering was performed on each chromosome $(n=2)$. B. Gene expression profile using RNA-seq analysis (GES131137). Genes whose expression was altered in OS5K cells were screened based on reads per kilobase of exon per million mapped reads (RPKM > 3 in 
either OSPa or OS5K cells and I Log2 (RPKM in OS5K/RPKM in OSPa) | 1 ). Then, the z-score was calculated using RPKM in each gene. Each gene expression profile was applied to hierarchical clustering. $(n=1)$. C. Correlation between DNA methylation and gene expression. Genes were screened by gene expression (RPKM in B, | Log2 (RPKM in OS5K/RPKM in OSPa) $\mid>1$ ) and DNA methylation score ( $\beta$-value in $A, \mid \beta$-value difference $\mid>0.1)$. The blue horizontal lines correspond to changes in gene expression more than twice, whereas the blue vertical lines correspond to an absolute difference in $\beta$-value of more than 0.1. D. Methylation status of the down-regulated genes. The top 12 decreased genes in OS5K cells were screened and compared with those in OSPa cells in B. Then, the methylation status was examined. Boxes represent the probes in their promoter $\mathrm{CpG}$ loci in A. Heatmap shows the gene expression in each cell or the $\beta$-value difference between OS5K and OSPa cells in each promoter CpG locus. E. Gene Ontology (GO) analysis of the hypermethylated genes. The top 300 hypermethylated genes in OS5K cells were screened and compared with those in OSPa cells in A. Then, their gene expression data in B were used for GO analysis. The graph shows the enriched GO terms which are down-regulated in OS5K cells with normalized enrichment scores (NES). 

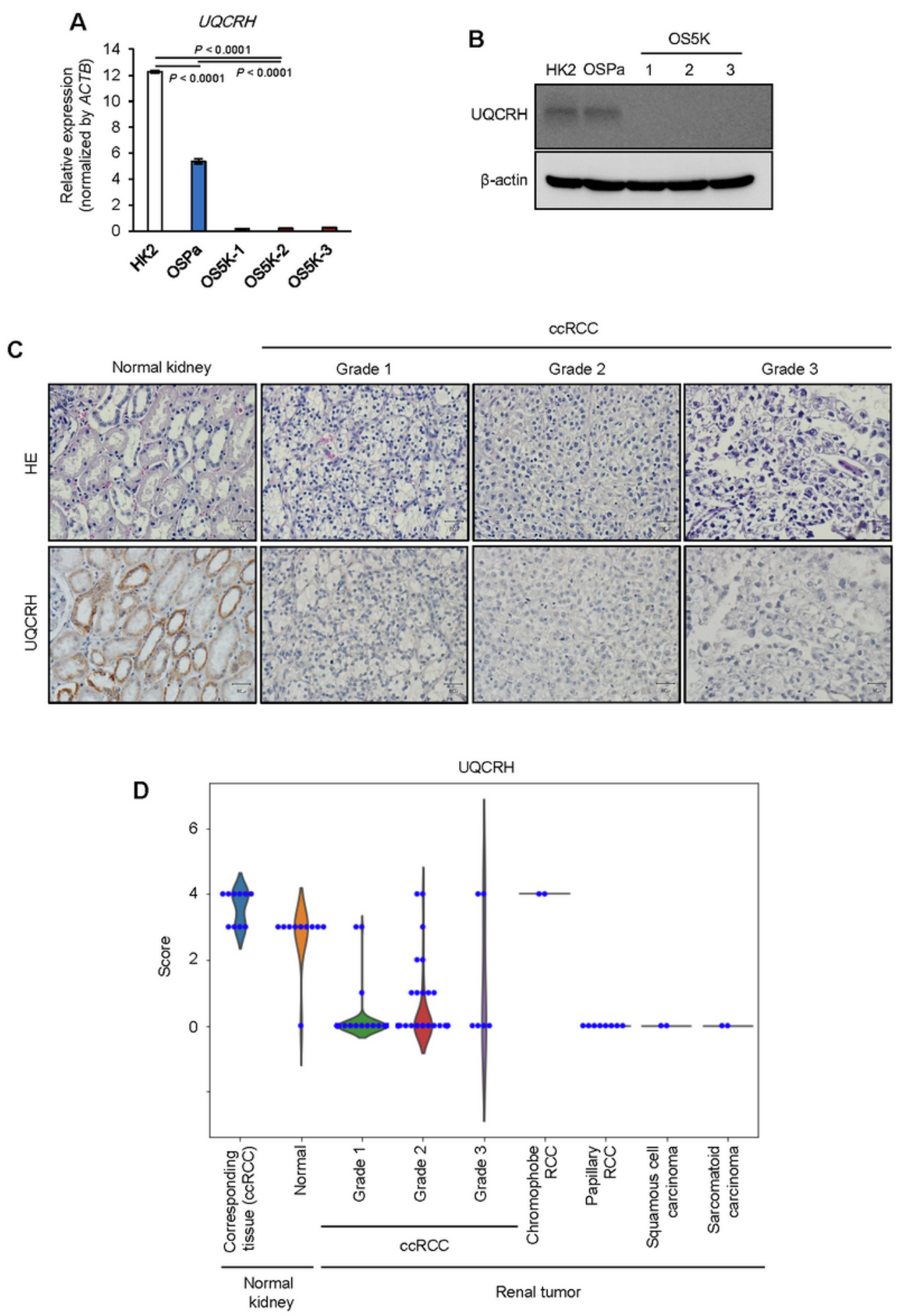

\section{Figure 4}

Expression of UQCRH is decreased in human renal tumor tissues. A, B. Expression of UQCRH in OS-RC-2 derivatives and HK-2 cells (control). UQCRH mRNA was analyzed using qRT-PCR analysis (A). Immunoblotting of UQCRH and $\beta$-actin (B). C, D. Immunohistochemical analysis of human normal renal tissues and renal tumor tissues. Tissue array was stained with HE (top) or anti-UQCRH antibodies (bottom). ( $n=100$, Grade 1 ccRCC; $n=52$, Grade 2; $n=6$, Grade 3; $n=10$, human normal kidney; $n=10$, 
normal adjacent tissue; $\mathrm{n}=8$, papillary RCC; $\mathrm{n}=2$, squamous cell carcinoma; $\mathrm{n}=2$, chromophobe cell carcinoma; and $n=2$, sarcomatoid carcinoma). Representative images of UQCRH expression in normal kidneys and ccRCC Grades 1-3 (C) and their quantification (D). Scale bar, $30 \mu \mathrm{m}$. UQCRH expression in the tissue array was scored as follows: negative $=1$; weak $=2$; moderate $=3$; and strong $=4$.

A
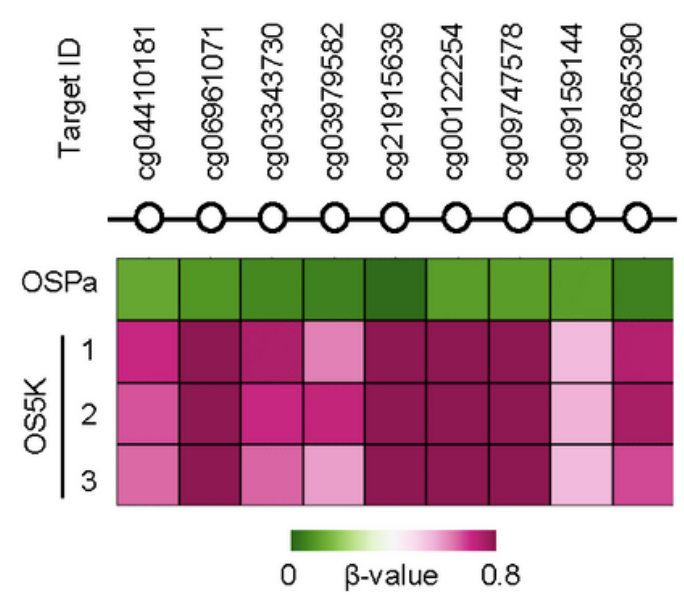

C

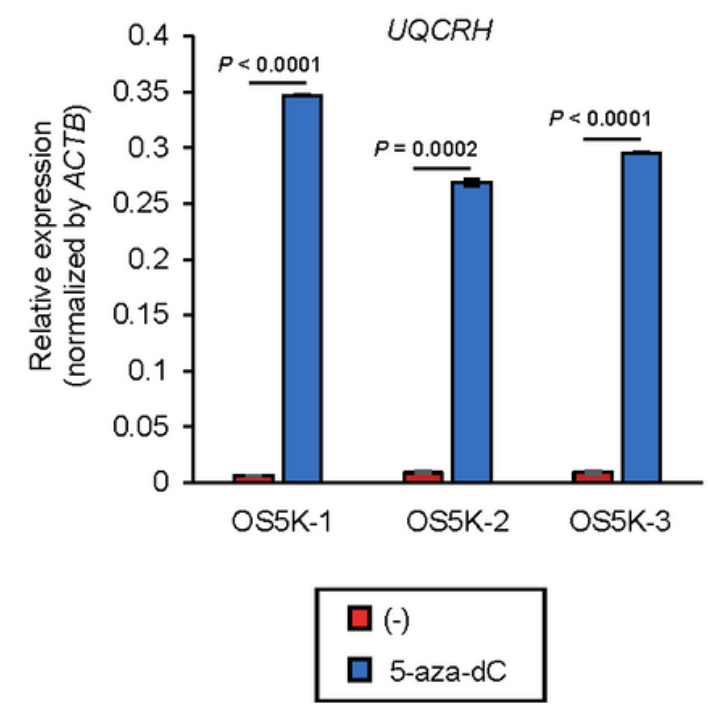

c
B
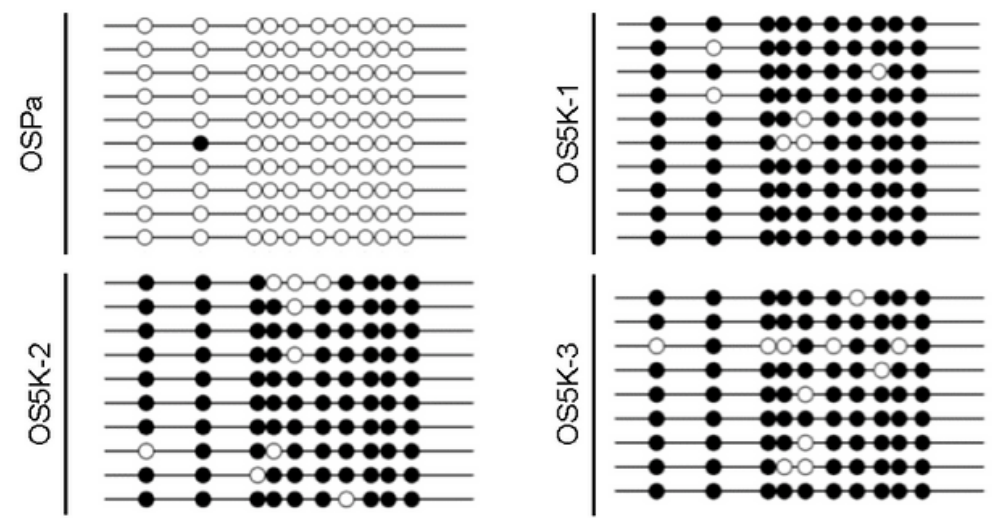

CPG site

Unmethylated

Mehylated

$\begin{array}{lllllll}-140 & -104 & -70 & -62 & -42 & -30\end{array}$

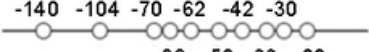

$\begin{array}{llll}-68 & -50 & -33 & -22\end{array}$

Figure 5

UQCRH expression is down-regulated by DNA methylation. A. Analysis of the methylation status of the UQCRH promoter region in OSPa, OS5K-1, OS5K-2, and OS5K-3 cells using the data set from the methylation array in Fig. 3B. Heatmap shows the $\beta$-value. B. Bisulfite-sequencing analysis of the UQCRH promoter region in OSPa, OS5K-1, OS5K-2, and OS5K-3 cells. Black circles mark the methylated CpG loci; white, unmethylated $(n=10$, OSPa; $n=10$, OS5K-1; $n=10$, OS5K-2; and $n=9$, OS5K-3. C, D. Upregulation of UQCRH in OS-RC-2 derivatives by the inhibition of DNMTs. OS5K cells were cultured with or without 5aza-dC $(0.3 \mu \mathrm{M})$ for $96 \mathrm{~h}$. UQCRH mRNA was analyzed by qRT-PCR analysis (C). The bars represent the 
mean \pm SD (two-sided Student's t-test; $n=2$ ). UQCRH protein and $\beta$-actin protein were detected using immunoblotting (D).
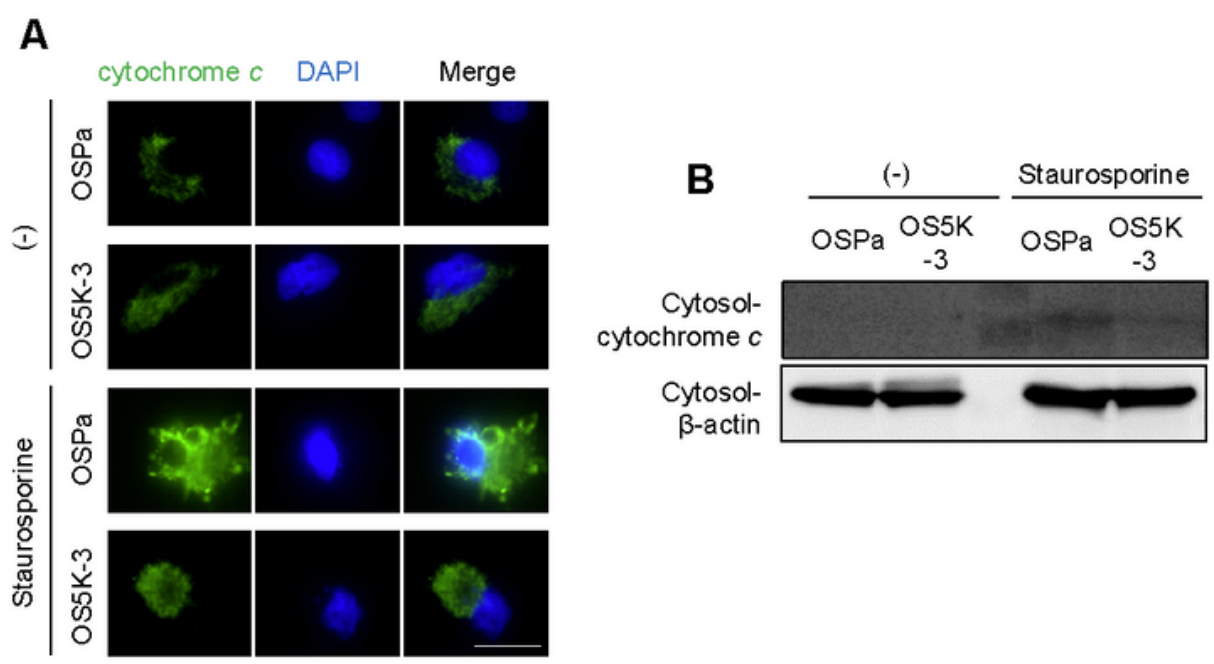

C
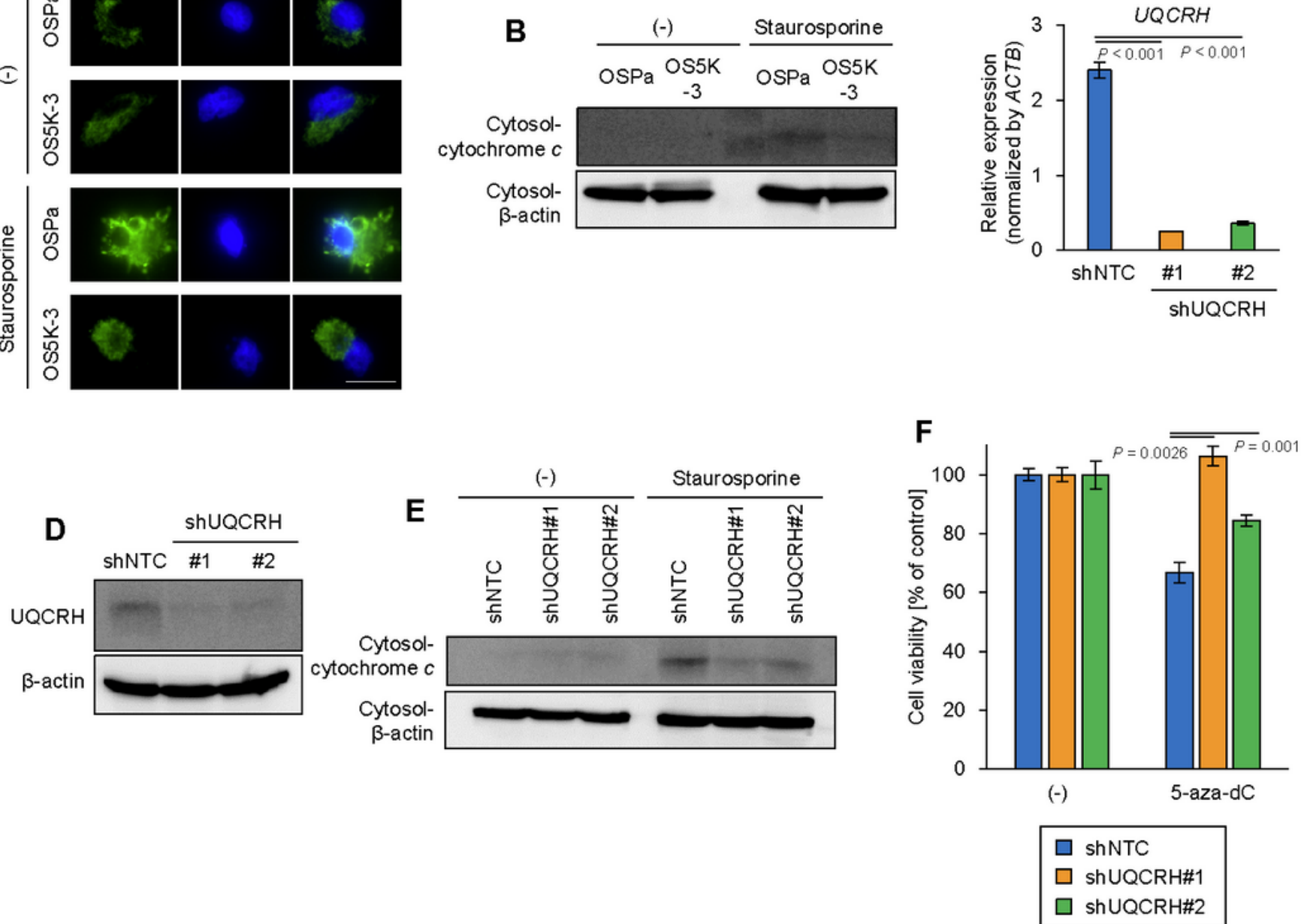

G
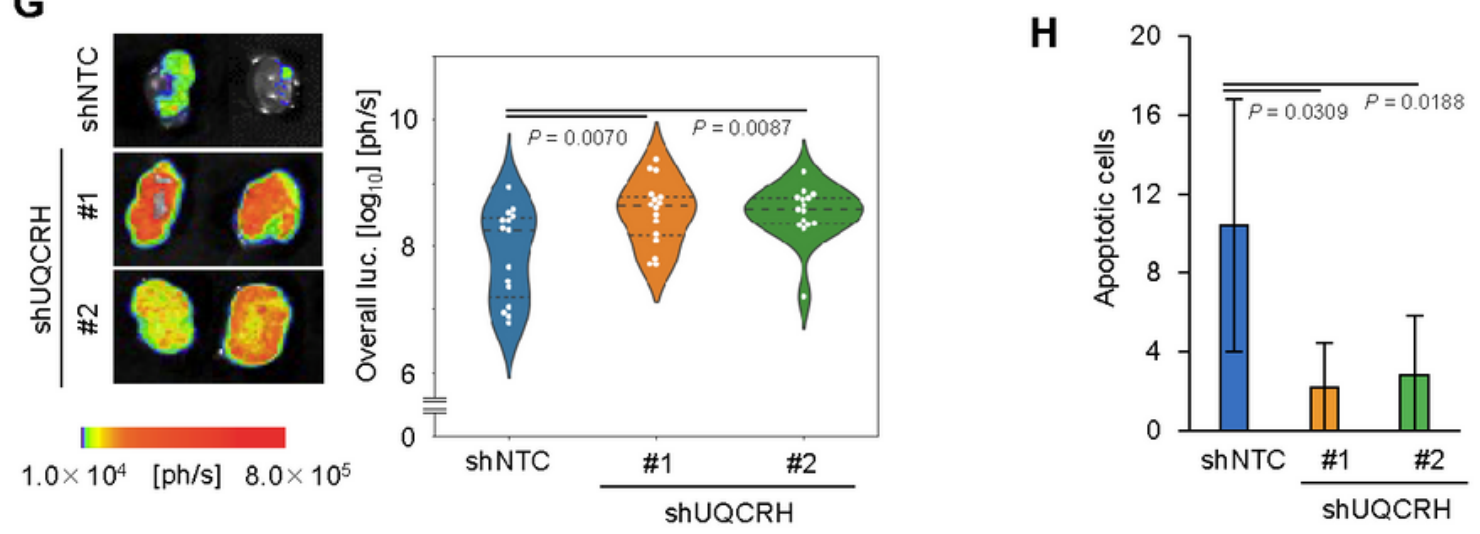

Figure 6

DNMT3B enhances apoptosis resistance by decreasing UQCRH in renal cancer cells. A. Immunocytochemical staining with cytochrome $c$ of OS-RC-2 derivatives. Cells were treated with staurosporine $(0.5 \mu \mathrm{M})$. Nucleus was stained with DAPI. Scale bars, $25 \mu \mathrm{m}$. B. Immunoblotting of 
cytochrome $c$ and $\beta$-actin in OS-RC-2 derivatives. Cells were treated with or without staurosporine $(1 \mu \mathrm{M})$ for $6 \mathrm{~h}$. Cytosolic fraction was collected. Molecular weight marker is indicated. C, D Knockdown of UQCRH in OSPa cells. OSPa cells were infected with lentiviral vectors encoding shNTC, shUQCRH\#1, or shUQCRH\#2. UQCRH mRNA was measured using qRT-PCR analysis (C). The bars represent the mean \pm SD (one-way ANOVA and Tukey's test, $n=2$ ). UQCRH protein was detected using immunoblotting (D). E. Immunoblotting of cytochrome $c$ and $\beta$-actin in ccRCC cells. Cells were treated with staurosporine $(1 \mu \mathrm{M})$ for $6 \mathrm{~h}$. Cytosolic fraction was collected. F. Cell proliferation assay of cCRCC cells. Cells were cultured with or without 5-aza-dC $(10 \mu \mathrm{M})$ for $96 \mathrm{~h}$. The bars represent the mean \pm SD (one-way ANOVA and Tukey's test, $n=3$ ). G. Representative images (left) of ex vivo bioluminescence imaging of the primary tumors. ccRCC cells were inoculated orthotopically. The mice were sacrificed 36 days after the inoculation. Panel shows the representative images of primary tumors. Quantification of (right). The bars represent the mean and 1st and 3rd quartile (one-way ANOVA and Tukey's test; $n=15$, shNTC; $n=16$, shUQCRH\#1; $n=$ 15, shUQCRH\#2). H. Apoptotic cells in primary tumor tissues from the experiments in G. Primary tumor tissues were subjected to TUNEL assay. Nucleus was stained with DAPI. Total apoptotic cells in the independent fields were quantified. The bars represent the mean \pm SD (one-way ANOVA and Tukey's test; $n=7$, shNTC; $n=6$, shUQCRH\#1; $n=6$, shUQCRH\#2). 
A
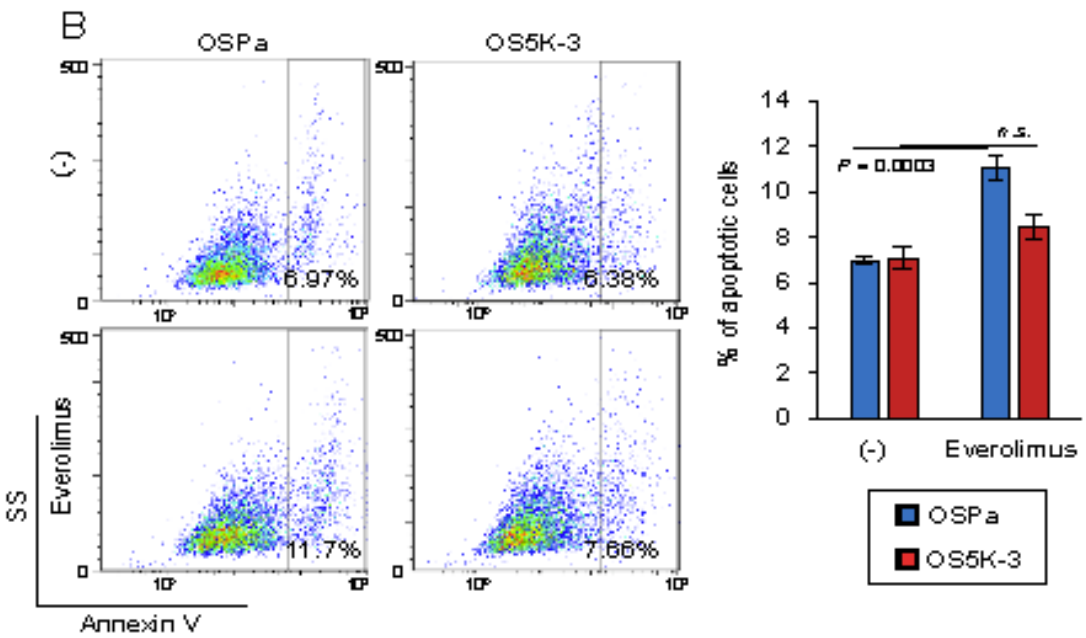

B
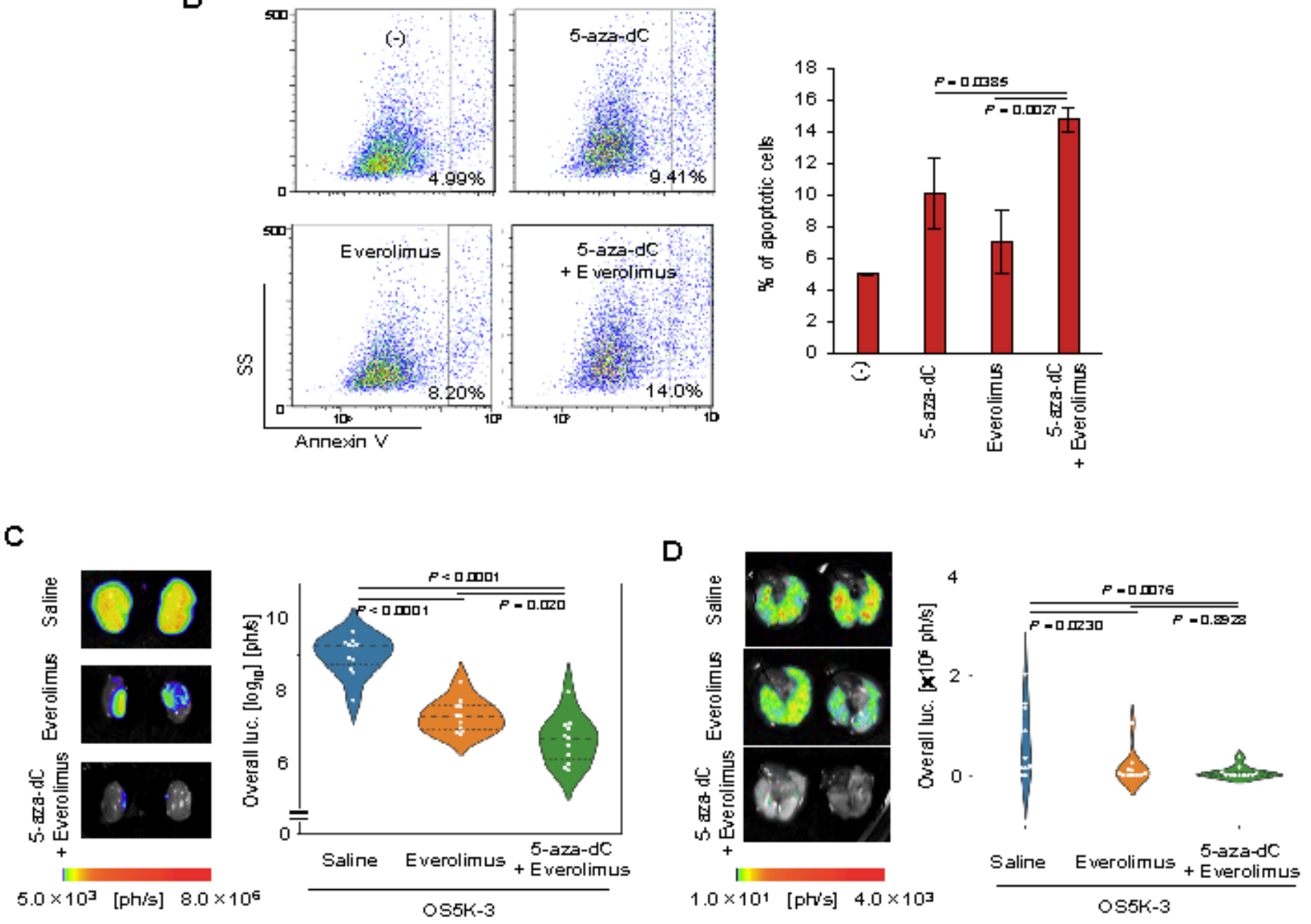

\section{Figure 7}

DNMT inhibitor enhances sensitivity to mTOR inhibitor in ccRCC cells. A. Apoptotic cell detection as determined using cytometric analysis of 0S5K-3 cells following everolimus treatment. Cells were treated with or without everolimus (3 $\mu \mathrm{M})$ for $48 \mathrm{~h}$. Representative panel (left); percentages of Annexin V+cell populations (right). The bars represent the mean \pm SD (two-sided Student's t-test; $n=3$ ). B. Apoptotic cell detection as determined using cytometric analysis of OS5K-3 cells following everolimus or 5-aza-dC 
treatment. Cells were treated with or without everolimus $(3 \mu \mathrm{M})$ for $24 \mathrm{~h}$ or 5 -aza-dC $(0.3 \mu \mathrm{M})$ for $72 \mathrm{~h}$. Representative panel (left) and percentages of Annexin $\mathrm{V}+$ cell populations (right). The bars represent the mean $\pm S D$ (one-way ANOVA and Dunnet's test; $n=3$ ) C, D. Ex vivo bioluminescence imaging of primary (C) and metastatic lung (D) tumors and their quantification. OS5K-3 cells were pre-treated with or without 5-aza-dC $(0.1 \mu \mathrm{M})$ for $72 \mathrm{~h}$ and inoculated orthotopically. The mice-bearing untreated cancer cells were administrated with saline or everolimus. The mice-bearing 5-aza-dC-treated cancer cells were administrated with everolimus. The mice were sacrificed 16 days after the inoculation. The bars represent the mean and 1st and 3rd quartiles (C: two-sided Welch's t-test; D: two-sided Student's t-test; $n=11$ each).

\section{Supplementary Files}

This is a list of supplementary files associated with this preprint. Click to download.

- MiyakuniSup.docx 